\title{
A rare case of acute pancreatitis induced by a gastrointestinal stromal tumor arising from the gastric fundus
}

An 82-year-old woman presented as an emergency with a 20-hour history of abdominal distension and acute epigastric pain radiating to her back. The pain was associated with nausea, vomiting, anorexia, and weight loss. Physical examination revealed midepigastric tenderness with a palpable mass. Pertinent laboratory values included a high white blood cell count of $21.5 \times 10^{9} / \mathrm{L}$, a C-reactive protein (CRP) level of $17.8 \mathrm{mg} / \mathrm{L}$, a serum amylase of 1200 units/L, and urinary amylase of 2000 units/L. An abdominal computed tomography (CT) scan showed a $4.0 \times$ $4.0 \times 8.0-\mathrm{cm}$ duodenal mass, but no regional lymph node or distant metastases ( Fig. 1). The diagnosis of pancreatitis was proposed.

An upper gastrointestinal endoscopy showed gastric mucosa along with a huge mass that was dangling into the duodenum ( Fig. 2). During surgery, a rounded mass was found that was originating from the submucous membrane of the gastric fundus. This was found to have prolapsed into the second part of the duodenum and to be obstructing the pylorus and pancreatic duct.

The patient underwent a complete resection of the tumor ( Fig.3). Pathologic examination of the resected tumor revealed a gastric fundal gastrointestinal stromal tumor (GIST) of low grade spindle-cell type. Immunohistochemical markers confirmed the diagnosis of a GIST (CD34, DOG-1, and Kit/CD117 were positive; desmin, SMA, and S100 were negative; the Ki-67 proliferation index was $<1 \%$ ( Fig.4). Because the low level of proliferative activity indicated a low risk of malignancy and recurrence, there was no indication for adjuvant therapy with imatinib. The patient's postoperative course was uneventful and she was discharged 10 days after surgery. She will continue to receive long-term follow-up.

GISTs initially presenting as acute pancreatitis are exceedingly rare: only two cases have been reported in the world literature $[1,2]$. In conclusion, we report an unusual case of 82-year-old woman who presented with acute pancreatitis that was found to be due to a GIST arising from the gastric fundus.

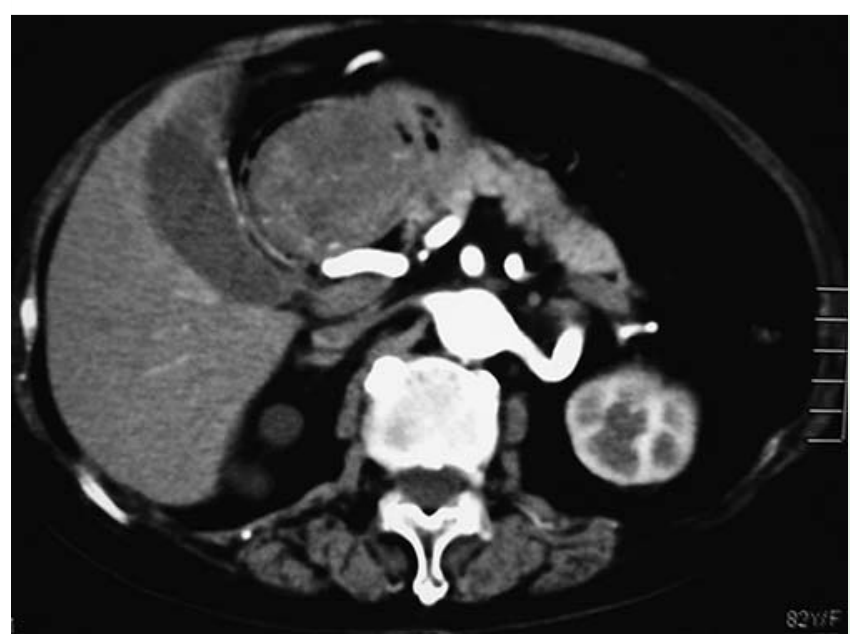

Fig. 1 Abdominal contrast-enhanced computed tomography (CT) scan showing a $4.0 \times 4.0 \times 8.0-\mathrm{cm}$ duodenal mass with heterogeneous contrast enhancement, as well as evidence of mild pancreatitis.
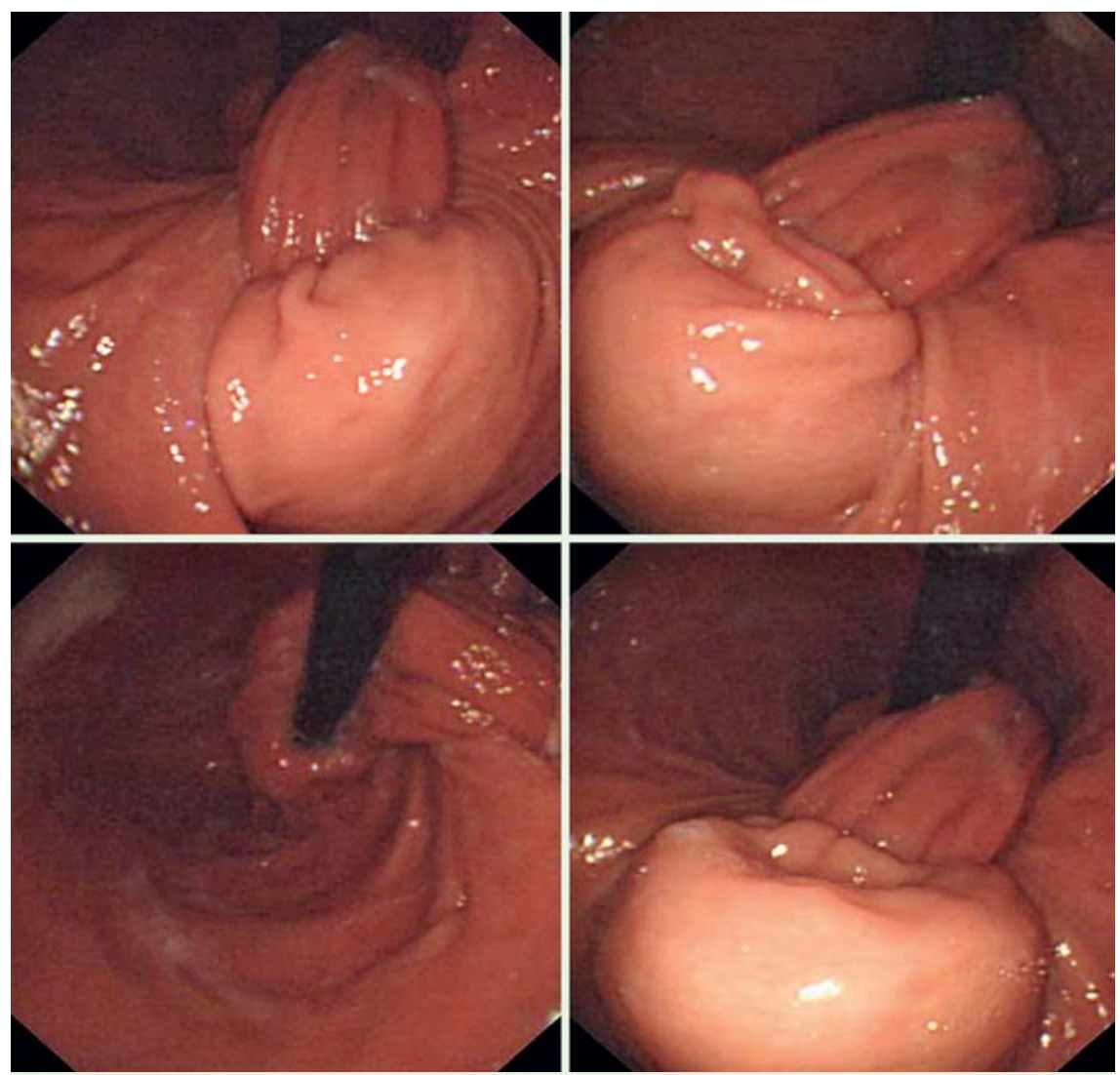

Fig.2 Images from an upper gastrointestinal endoscopy showing gastric mucosa along with a huge mass that was dangling into the duodenum. 


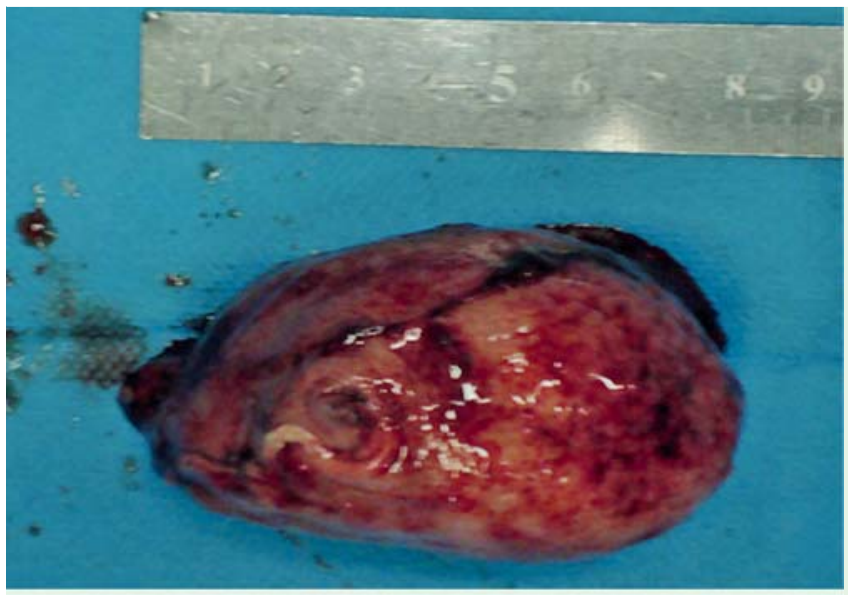

Fig. 3 Photographs of the surgical specimen, which contained a $4 \times 8$-cm tumor.
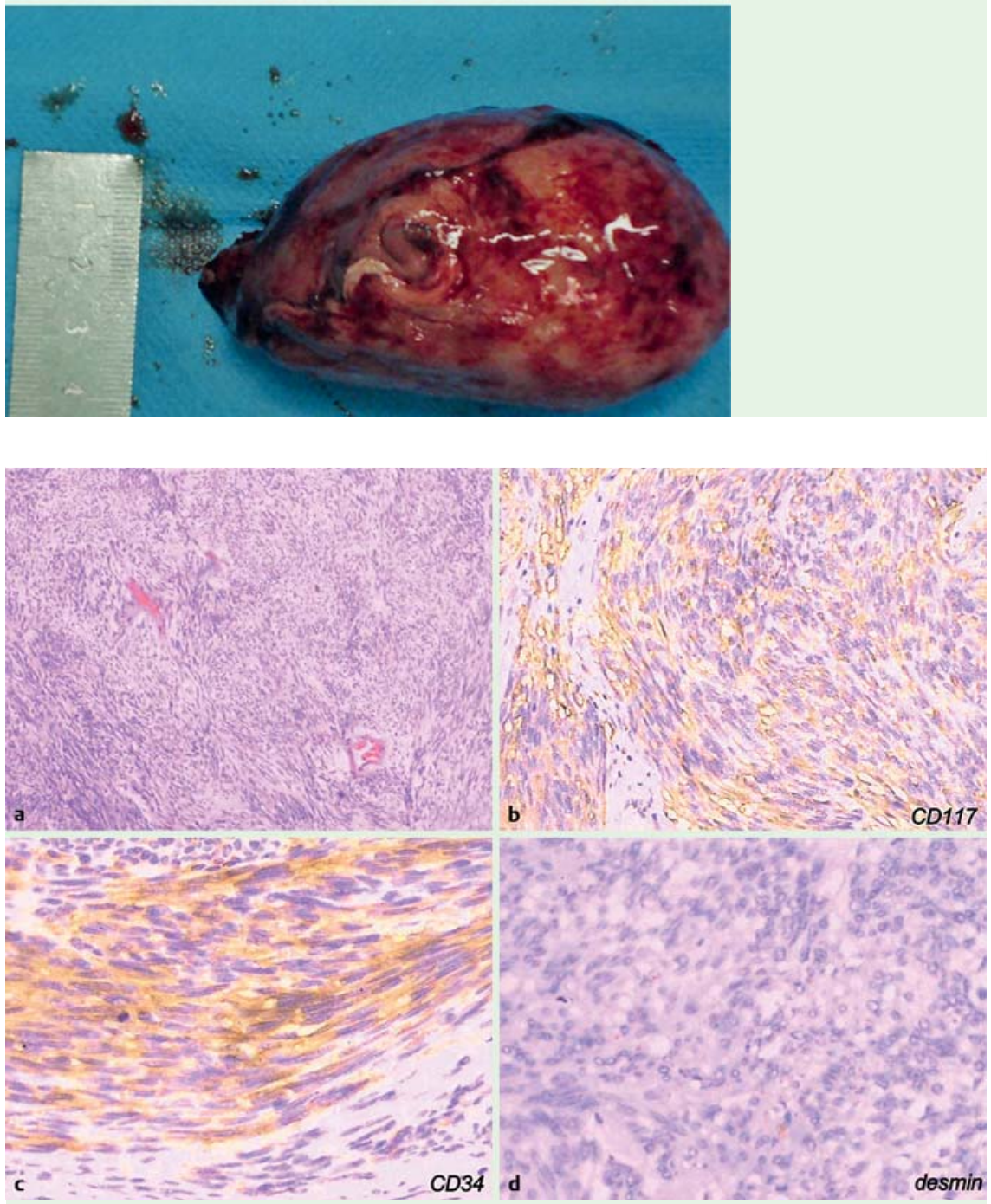

Fig. 4 Histologic appearances of the resected gastrointestinal stromal tumor (GIST). a Staining with hematoxylin and eosin (H\&E) revealed evidence of whirling sheets of spindle cells with a low level of pleomorphism and mitotic activity (2 mitoses/50 high-power fields [HPF]) and occasional evidence of necrosis. $\mathbf{b}$ - $\mathbf{d}$ Immunohistochemical staining for: $\mathbf{b}$ CD117 was positive; $\mathbf{C} C D 34$ was positive; $\mathbf{d}$ desmin was negative.
Endoscopy_UCTN_Code_CCL_1AB_2AD_3AB

\section{Competing interests: None}

\section{J. Sun ${ }^{1}$, X. Shen ${ }^{1}$, Z. Li ${ }^{2}$, J. Zhu ${ }^{1}$}

${ }^{1}$ Department of Gastroenterology, Jinan Central Hospital, Shandong University, Jinan, China

2 Department of Gastroenterology, Changhai Hospital, Second Military Medical University, Shanghai, China

\section{References}

1 Soufi M, Chad B. Stromal duodenal tumor revealed by an acute pancreatitis: report of a case. J Gastrointest Cancer 2010; 41: 88-91

2 Senadhi V, Arora D, Jani N. Gastrointestinal stromal tumor (GIST) presenting with acute pancreatitis. Endoscopy 2011; 43: E76

\section{Bibliography}

DOI http://dx.doi.org/

$10.1055 / \mathrm{s}-0032-1325863$

Endoscopy 2012; 44: E426-E427

(c) Georg Thieme Verlag KG

Stuttgart · New York

ISSN 0013-726X

\section{Corresponding author}

\section{J. Zhu, MD}

Jinan Central Hospital, Shandong University

105 Jiefang Road

Jinan 250012

China

Fax: +86-531-86942457

sjbzjy@163.com 